\title{
Huesos Suturales Umbilicados
}

\author{
Umbilicated Sutural Bones
}

\author{
Juan Silva* \& Claudia Araya*
}

SILVA, J. \& ARAYA, C. Huesos suturales umbilicados. Int. J. Morphol., 34(2):616-619, 2016.

RESUMEN: Los huesos suturales son huesos supernumerarios observables en suturas y fontanelas del cráneo, variables en su número, forma, tamaño y posición. Formados desde centros de osificación normales y/o adicionales. Calleja en 1870, describe un hueso sutural en forma de anillo con un centro óseo (umbilicados). Nuestro objetivo de investigación, fue buscar y describir estos huesos. Se utilizaron 71 cráneos de adultos chilenos de ambos sexos, en los cuales se buscó, caracterizó, fotografió y midió a los huesos suturales umbilicados. Se hallaron dos cráneos con presencia de estos huesos, representando el 2,82\% del total de la muestra y el $8 \%$ de los cráneos con huesos suturales. Los resultados concuerdan con la bibliografía, en cuanto a presencia de huesos suturales, así como su relación directa con el sexo y el tamaño del cráneo. También pudimos comprobar la observación y descripción de Calleja, encontrando huesos suturales que cumplían con su descripción. El conocimiento de estos huesos es de utilidad en la identificación médico legal.

PALABRAS CLAVE: Huesos suturales; Huesos wormianos; Huesos del cráneo; Suturas y fontanelas del cráneo; Identificación médico legal.

\section{INTRODUCCIÓN}

Los huesos suturales (wormianos) corresponden a huesos planos, supernumerarios, irregulares, inconstantes e independientes, que son posibles de observar, de manera accidental, entre las suturas y fontanelas del cráneo (Strambio, 1854; Poirier et al., 1908; Orts Llorca, 1959; Testut-Latarjet, 1965; Aprile et al., 1967; Prives et al., 1984; Latarjet \& Ruiz Liard, 2010; Murlinmanju et al., 2011). Estas piezas óseas son muy variables en su número, forma, tamaño y posición (Cruveilhier, 1851; Sappey, 1874; Aprile et al.; Williams, 1998; Garcia-Hernández et al., 2009). Están formados desde centros de osificación normales y/o adicionales (Cruveilhier; Orts Llorca; Lockhart et al., 1988; Williams; Garcia-Hernández et al., 2007) y son considerados de importancia genética y hereditaria, relacionados con variables étnicas (Garcia-Hernández et al., 2009), además de ser importantes en anatomía humana, antropología física, imagenología e identificación médico legal.

Estos huesos se clasifican en verdaderos (aquellos provenientes de centros de osificación adicionales) y falsos (aquellos provenientes de centros de osificación normales y que han permanecido independientes), tanto de origen membranoso como de origen cartilaginoso. A su vez se pueden subclasificar según la posición que ocupen en el cráneo. Así se pueden observar fontanelares, suturales e insulares (Poirier et al.; Orts Llorca; Testut \& Latarjet, 1965;
Aprile et al:; Rouviére \& Delmas, 1999; Latarjet \& Ruiz Liard, 2010).

Julián Calleja en su Tratado de Anatomía Humana (Calleja y Sánchez, 1870) publica que Juan Fourquet observó "un hueso wormiano al parecer desarrollado por 2 puntos, uno central y otro periférico, que dieron por resultado la construcción de una pieza, compuesta de un círculo perforado a manera de diafragma óptico, cuya perforación se encontraba ocupada por un punto óseo central". Este hueso sutural, con estas características, no vuelve a estar presente en la bibliografía y no está incluido dentro de las clasificaciones de estos huesos.

Este hueso sutural descrito por Calleja y Sánchez, no debe confundirse con los huesos suturales insulares, que se describen como huesos que se desarrollan lejos de las suturas y fontanelas del cráneo, en medio de algunos huesos planos, por su cara endocraneal (Poirier et al.; Testut \& Latarjet; Rouviére \& Delmas; Latarjet \& Ruiz Liard).

Esta discrepancia entre lo descrito por Calleja y Sánchez y los otros autores tradicionales determinó el estudio que se presenta a continuación y que tuvo como objetivo la búsqueda y descripción de dichos huesos en cráneos humanos. Debido a su forma característica, en este trabajo, se decidió llamarlos huesos suturales umbilicados. 


\section{MATERIAL Y MÉTODO}

Se analizaron 71 cráneos de individuos adultos chilenos de ambos sexos, pertenecientes a la osteoteca de la Escuela de Medicina de la Universidad San Sebastian, en búsqueda de huesos suturales umbilicados. De los huesos suturales encontrados, se consideró su clasificación, localización, posición, forma, tamaño y cantidad de "ombligos óseos" que presentaban.

Los cráneos se fotografiaron con cámara digital marca Panasonic $^{\oplus}$ modelo DMC-GX1. Luego se procedió a su medición con pelvímetro marca $\operatorname{Martin}^{\odot}$ y cinta antropométrica marca Rosscraft ${ }^{\odot}$. Los huesos suturales umbilicados se midieron con caliper digital marca Mitutoyo@ serie 500 modelo 144 .

\section{RESULTADOS}

Del total de 71 cráneos de la muestra, 25 presentaron huesos suturales $(35,2 \%)$. De estos últimos, se encontraron

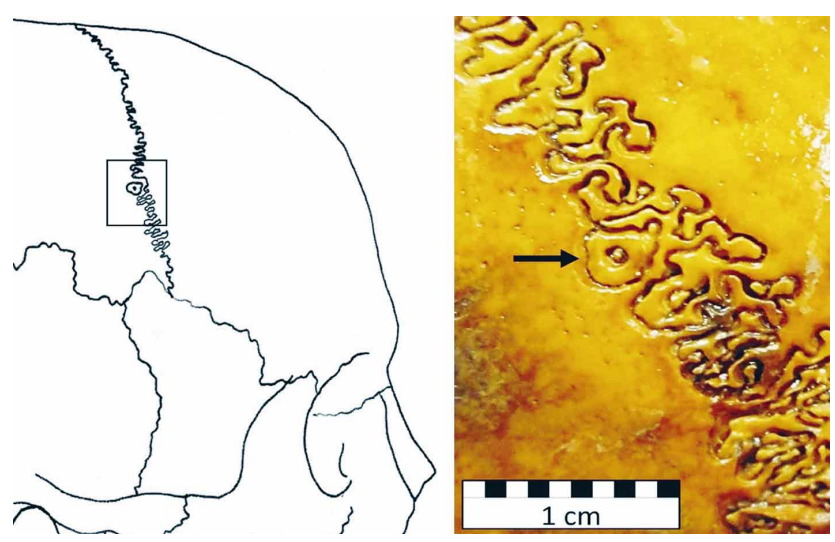

Fig. 1. Hueso sutural umbilicado en el costado derecho de la sutura coronal del cráneo $\mathrm{n}^{\circ} 9$, en este hueso se puede observar un "ombligo óseo" (flecha). dos cráneos que presentaban huesos suturales umbilicados, el cráneo identificado como "9" y el cráneo identificado como " 15 ", representando el $2,82 \%$ del total de la muestra y el $8 \%$ del total de cráneos con presencia de huesos suturales.

El cráneo $\mathrm{n}^{\circ} 9$, perteneció a un individuo de sexo femenino con un rango etario entre 20 a 30 años, de proporciones normales (diámetro cefálico anteroposterior máximo: $192 \mathrm{~mm}$, diámetro cefálico transverso máximo: $171 \mathrm{~mm}$ y circunferencia máxima: $476 \mathrm{~mm}$ ), sin variantes anatómicas importantes. En este cráneo se encontró un hueso sutural verdadero, posible de ser clasificado como umbilicado, con una forma de triángulo irregular ubicado en la sutura coronal del lado derecho en un punto medio entre bregma y pterion, con 3,88 $\mathrm{mm}$ de largo y $3,08 \mathrm{~mm}$ de ancho. Este hueso presentó un pequeño "ombligo óseo" de posición central, solo observable en la cara exocraneal de la sutura (Fig. 1).

El cráneo $\mathrm{n}^{\circ} 15$, perteneció a un individuo de sexo masculino con un rango etario entre 30 a 40 años, de proporciones mayores al cráneo $\mathrm{n}^{\circ} 9$ (diámetro cefálico anteroposterior máximo: $225 \mathrm{~mm}$, diámetro cefálico transverso máximo: $195 \mathrm{~mm}$ y circunferencia máxima: 563 $\mathrm{mm}$ ), con presencia de la sutura frontal persistente y huesos suturales en la sutura escamosa y en la lambdoidea, además de huesos suturales en posición lambda y asterion. Este cráneo presentó tres huesos suturales posibles de clasificar como umbilicados, todos presentes en la sutura lambdoidea. El primer hueso sutural umbilicado ubicado en la sutura lambdoidea izquierda en un punto medio entre lambda y asterion presentó un tamaño de 23,04 mm de largo y 17,25 $\mathrm{mm}$ de ancho, con presencia de dos "ombligos óseos", ambos en cercanía del borde, uno en posición ínfero lateral y de forma ovoidea de 1,66 $\mathrm{mm}$ de diámetro. El otro se ubica en posición supero lateral y presenta forma ovoidea con un largo de $1,59 \mathrm{~mm}$ y un ancho de $0,85 \mathrm{~mm}$, ambos solo observables en la cara exocraneal (Fig. 2). El segundo hueso sutural umbilicado ubicado en la sutura lambdoidea izquierda inmediatamente lateral a lambda presentó una forma irre-

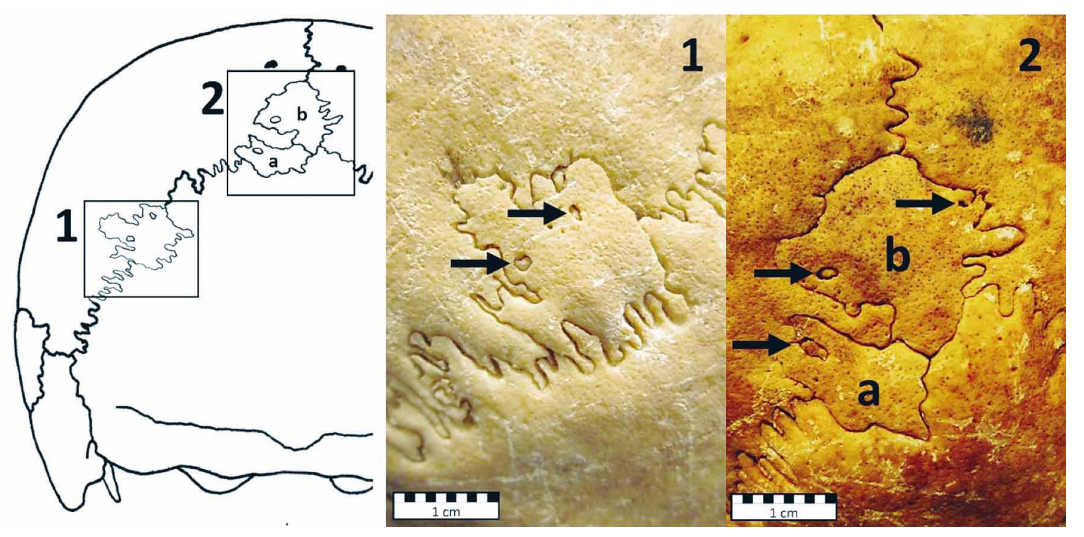

Fig. 2. Huesos suturales umbilicados del cráneo $n^{\circ} 15$. 1) Hueso sutural umbilicado en sutura lambdoidea que presenta 2 "ombligos óseos" (flechas). Hueso sutural umbilicado inmediatamente lateral a lambda (a), que presenta 1 "ombligo óseo" (flecha). Hueso sutural en posición lambda (b), que presenta dos "ombligos óseos". 
gularmente cuadrangular de 20,53 $\mathrm{mm}$ de largo y $12,42 \mathrm{~mm}$ de ancho con presencia de un "ombligo óseo" cerca de su extremo súpero lateral, de forma ovoidea con un largo de $3,61 \mathrm{~mm}$ y un ancho de $1,75 \mathrm{~mm}$, solo observable en su cara exocraneal (Fig. 2). El tercer hueso sutural umbilicado ubicado en posición lambda (fontanelar), presentó una forma irregularmente romboidal con un tamaño de $23,31 \mathrm{~mm}$ de altura y $28,95 \mathrm{~mm}$ de ancho. Presentó dos "ombligos óseos" el primero de ellos se observa en el ángulo lateral izquierdo, posee forma ovoidea y presentó un tamaño de $2,82 \mathrm{~mm}$ de largo y de 1,56 de ancho. El segundo de estos "ombligos óseos" se ubicó en el ángulo lateral derecho, de forma ovoidea, con un largo $1,46 \mathrm{~mm}$ y un ancho de $0,65 \mathrm{~mm}$, solo observable en su cara exocraneal (Fig. 2).

\section{DISCUSION}

En nuestro estudio, de un conjunto de 71 cráneos estudiados, encontramos 25 que presentaban algún tipo de hueso sutural, ya sea de posición sutural o fontanelar. La presencia de huesos suturales en la muestra $(35,2 \%)$ es menor a la encontrada por Murlimanju $(73,1 \%)$ pero mayor a la hallada por Braga et al. (2000) (15,1\%).

Encontramos concordancia con Sappey y Orts Llorca, observando un mayor número de huesos suturales en el cráneo de dimensiones mayores que en aquel de dimensiones menores. A su vez, también pudimos constatar, en forma coincidente con lo descrito por Orts Llorca y Aprile et al., una mayor cantidad de huesos suturales en el cráneo de sexo masculino versus el femenino.

Dentro de los huesos suturales presentes en la muestra, encontramos dos huesos que coinciden con la descripción realizada en 1870 por Calleja y Fourquet representando el 2,82 \% del total de la muestra. Estos huesos suturales que hemos denominado "umbilicados", presentaban características semejantes a las descritas por estos anatomistas españoles.

Las características de estos huesos corresponden a un hueso sutural con forma de triángulo, cuadrado o rombo irregular, perforado, cuya cavidad se encuentra ocupada por un punto óseo central, que en este trabajo hemos denominado "ombligo óseo".

Además, en la muestra, los huesos suturales umbilicados están presentes tanto en la sutura coronal como en la sutura lambdoidea y se pueden observar en posición sutural como fontanelar, no pudiendo determinar ninguna tendencia en su posición. Adicionalmente, en la muestra, estos huesos y sus ombligos, son variables en su posición, forma y cantidad.

La morfología característica de estos huesos suturales umbilicados está relacionada con la formación de la propia sutura dentada. Posiblemente, el ombligo óseo corresponde a un "diente" de uno de los huesos que forma la sutura, que ha quedado rodeado por dos "dientes" del hueso vecino, en que se ha producido la fusión de los extremos libres de dichos "dientes", encerrando en un círculo óseo al extremo libre del "diente prisionero".

El reconocimiento de estas particulares estructuras anatómicas, evidenciables y registrables a través de la imagenología médica, permite individualizar a los individuos y ser utilizadas en identificación médico legal humana, debido a sus características de inmutabilidad, particularidad y relativa perennidad. Esta especial condición y utilidad, debe ser considerada, para incorporar a la clasificación actual de los huesos suturales, a los umbilicados.

SILVA, J. \& ARAYA, C. Umbilicated sutural bones. Int. J. Morphol., 34(2):610-615, 2016.

SUMMARY: The sutural bones are visible supernumerary bones in skull sutures and fontanelle, and are variable in number, shape, size and position. Formed from normal and /or additional ossification, Calleja in 1870, describes a sutural ring-shaped bone with a bone center (umbilicated). Our research objective was to look for and describe these bones. We used 71 skulls of Chilean adults of both sexes, in which we sought, marked, photographed and measured the molluscum sutural bones. Two (2) skulls were found with presence of these bones representing $2.82 \%$ of the total sample and $8 \%$ sutural skulls with bones. The results are consistent with the literature regarding presence of sutural bones, and their direct relationship to sex and size of the skull. We also noted the observation and description by Calleja, finding sutural bones that met their description. Knowledge of these bones is useful in forensic identification.

KEY WORDS: Sutural bones; Wormian bones; Skull bones; Sutures and fontanelles of the skull; Forensic identification.

\section{REFERENCIAS BIBLIOGRÁFICAS}

Aprile, H.; Figún, M. E. \& Garino, R. R. Anatomía Odontológica Orocervicofacial. Buenos Aires, El Ateneo, 1967.

Braga, M. T. T.; Gabrielli, C.; De Souza, A.; Rodrigues, C. F. S. \& Marino, J. C. Huesos suturales en el pterion. Rev. Chil. Anat., 18(1):97-101, 2000. 
SILVA, J. \& ARAYA, C. Huesos suturales umbilicados. Int. J. Morphol., 34(2):616-619, 2016.

Calleja y Sánchez, J. Tratado de Anatomía Humana. Madrid, Establecimiento Tipográfico de R. Labajos, 1870.

Cruveilhier, J. Traité d'Anatomie Descriptive. Paris, Labé, 1851.

García-Hernández, F. \& Murphy-Echeverría, G. Frecuency of wormian lambdoid bone in skulls with artificial deformation in Northern Chile. Int. J. Morphol., 27(3):933-8, 2009.

García-Hernández, F.; Díaz Blanco, J. L. \& Murphy Echeverría, G. T. Incidence of sutural bones in a population of chango skulls of Northern Chile, Antofagasta. Int. J. Morphol., 25(4):801-4, 2007.

Latarjet, M. \& Ruiz Liard, A. Anatomía Humana. Buenos Aires, Editorial Médica Panamericana, 2010.

Lockhart, R. D.; Hamilton, G. F. \& Fyfe, F. W. Anatomía Humana. Ciudad de México, Interamericana, 1988.

Murlimanju, B. V.; Prabhu, L. V.; Ashraf, C. M.; Kumar, C. G.; Rai, R. \& Maheshwari, C. Morphological and topographical study of Wormian bones in cadaver dry skulls. J. Morphol. Sci., 28(3):176-9, 2011.

Orts Llorca, F. Anatomía Humana. Barcelona, Científico-Medica, 1959.

Poirier, P.; Charpy, A. \& Cuneo, B. Tratado Elemental de Anatomía Humana. Madrid, Imprenta y Libreria de Nicolás Moya, 1906.

Prives, M.; Lisenkov, N. \& Bushkovich, V. Anatomía Humana. Moscú, MIR, 1984.

Rouviére, H. \& Delmas, A. Anatomía Humana Descriptiva, Topográfica y Funcional. Barcelona, Masson, 1999.

Sappey, P. C. Tratado de Anatomía Descriptiva. Madrid, Carlos Bailly Bailliere, 1874.

Strambio, G. Elementi di Anatomia Umana Descrittiva, Topografica e Generale. Milano, Francesco Vallardi, 1854.

Testut, L. \& Latarjet, A. Tratado de Anatomía Humana. Barcelona, Salvat Editores, 1965.

Williams, P. Anatomía de Gray: Bases Anatómicas de la Medicina y la Cirugía. Madrid, Harcourt Brace, 1998.

\author{
Dirección para Correspondencia: \\ Prof. Dr. Juan Luis Silva R. \\ Facultad de Medicina \\ Universidad San Sebastian \\ Providencia, Santiago \\ CHILE \\ Email: juan.silva@uss.cl
}

Recibido: 28-10-2015

Aceptado: 06-04-2016 